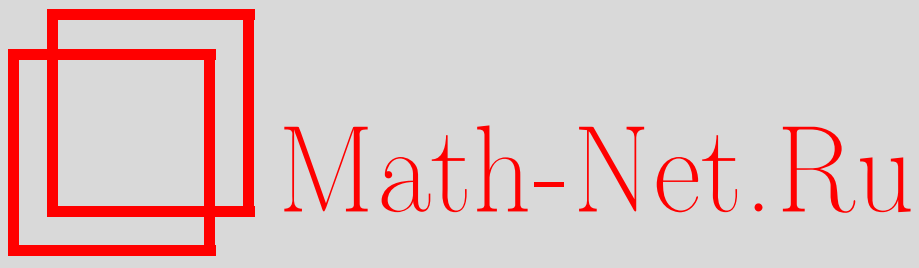

Д. Н. Туляков, О локальной асимптотике отношения ортогональных полиномов в окрестности крайней точки носителя меры ортогональности, Матем. сб., 2001, том 192, номер 2, 139-160

DOI: https://doi.org/10.4213/sm547

Использование Общероссийского математического портала Math-Net.Ru подразумевает, что вы прочитали и согласны с пользовательским соглашением

http://www . mathnet.ru/rus/agreement

Параметры загрузки:

IP : 54.205 .225 .156

26 апреля 2023 г., 13:36:58 


\title{
Д.Н. Туляков \\ О локальной асимптотике отношения ортогональных полиномов в окрестности крайней точки носителя меры ортогональности
}

\begin{abstract}
Предложен новый методисследования асимптотики ортогональных многочленов в окрестности крайней точки носителя меры ортогональности. Асимптотика в окрестности крайней точки определяется по асимптотике многочленов в самой точке и асимптотике рекуррентных коэффициентов. Рассмотрены применения полученного результата.

Библиографоя: 4 названия.
\end{abstract}

\section{§1. Введение}

Пусть последовательность полиномов $q_{n}(x)$ определяется рекуррентным соотношением

$$
q_{n+1}(x)=\left(x-b_{n}\right) q_{n}(x)-\Delta_{n} q_{n-1}(x) \quad(n \in \mathbb{N}), \quad q_{0}=1, \quad q_{1}=x-b_{0} .
$$

В работе исследуется асимптотическое поведение полиномов $q_{n}(x), n \rightarrow \infty$, в окрестности точки $x_{0}$. Эта задача является частью общей задачи об асимптотике ортогональных многочленов. Известно, что если точка $x_{0}$ находится вне носителя меры ортогональности, то последовательность $q_{n}(x), n \rightarrow \infty$, в окрестности точки $x_{0}$ быстро растет: в компактном случае эта последовательность асимптотически ведет себя как геометрическая прогрессия, в некомпактном случае порядок роста еше выше. Более сложной и важной для приложений является задача об асимптотике ортогональњых многочленов на носителе меры ортогональности и особенно в крайних точках сушественной части носителя. Положение крайних точек определяется асимптотикой рекуррентных коэффициентов (теорема Вейля об инвариантности сушественного спектра при компактных возмушениях). Решение задачи о локальной асимптотике зависит тогда от поведения многочленов в самой крайней точке. Приведем теперь примеры известных результатов в данном направлении.

1) Классическая формула Гейне-Мелера для полиномов Якоби описывает их асимптотическое поведение в окрестности точки 1:

$$
\lim _{n \rightarrow \infty} n^{-\alpha} P_{n}^{(\alpha, \beta)}\left(1-\frac{z^{2}}{2 n^{2}}\right)=J_{\alpha}(z)\left(\frac{z}{2}\right)^{-\alpha}, \quad P_{n}^{(\alpha, \beta)}(1)=\left(\begin{array}{c}
n+\alpha \\
n
\end{array}\right)
$$

Работа выполнена при поддержке программы "Ведущие научные школы" (грант № 00-1596132) и Российского фонда фундаментальных исследований (грант № 99-01-01251).

(C) Д.н. Туляков 2001 
2) Пример решения задачи в целом (в некоторой обшности), взятый из работы [1] без сохранения обозначений:

Теорема А (А.И. Аптекарев). Пусть $\left\{Q_{n}(x)\right\}-$ О.Н.С. многочленов по мере $d \mu \in M$, где класс $M$ определяется поведением рекуррентных коэффициентов системь $\left\{Q_{n}\right\}$ :

$$
a_{n} q_{n+1}(x)+b_{n} q_{n}(x)+a_{n-1} q_{n-1}(x)=x q_{n}(x), \quad a_{n} \rightarrow \frac{1}{2}, \quad b_{n} \rightarrow 0,
$$

u nycmb

$$
\frac{Q_{n+1}(1)}{Q_{n}(1)}=1+\frac{\alpha+\frac{1}{2}}{n}+O\left(n^{-1-\varepsilon}\right), \quad \alpha>-1, \quad \varepsilon>0 .
$$

Тогда при $n \rightarrow \infty$ равномерно на компактах комплексной $z$-плоскости

$$
n^{-(\alpha+1 / 2)} Q_{n}\left(1-\frac{z^{2}}{2 n^{2}}\right)=\frac{J_{\alpha}(z)}{z^{\alpha}}+o(1) .
$$

Теперь сформулируем и обсудим основной результат настоящей работы. Понятно, что без ограничения обшности достаточно рассмотреть $x_{0}=0$.

ТЕОРема 1. Пусть последовательность полиномов $\left\{q_{n}(x)\right\}_{n=0}^{\infty}$ удовлетворяет рекуррентным соотношениям

$$
q_{n+1}(x)=\left(x-b_{n}\right) q_{n}(x)-\Delta_{n} q_{n-1}(x) \quad(n \in \mathbb{N}), \quad q_{0}=1, \quad q_{1}=x-b_{0},
$$

с условием на коэффициенты:

$$
b_{n}=c n^{2 \beta}(1+o(1)) \quad \text { npu } \quad n \rightarrow \infty ; \quad c \neq 0,
$$

и пусть дополнительно известно, что последовательность значений в нуле $\left\{q_{n}(0)\right\}_{n=0}^{\infty}$ удовлетворяет условию

$$
-\frac{b_{n} q_{n}(0)}{q_{n+1}(0)}=2-\frac{2 \alpha+1}{n}+o\left(\frac{1}{n}\right) .
$$

Если параметры $\alpha$ и $\beta$ удовлетворяют неравенствам

$$
\beta<1, \quad \alpha>\beta-1
$$

то для отношения полиномов вблизи точки 0 можсно написать представление

$$
\frac{q_{N+1}\left(c N^{2 \beta-2} z\right)}{q_{N}\left(c N^{2 \beta-2} z\right)}=\frac{q_{N+1}(0)}{q_{N}(0)}\left(1+\frac{1}{N} f_{N}(z)\right),
$$

и в этом представлении имеется сходимость $f_{N}(z) \rightarrow f(z)$ в сферической метрике равномерно на компактах $K \subset \mathbb{C}$. Функиия $f(z)$ имеет явный вид:

$$
f(z)=\sqrt{2 z} J_{\frac{\alpha}{1-\beta}+1}\left(\frac{\sqrt{2 z}}{1-\beta}\right) / J_{\frac{\alpha}{1-\beta}}\left(\frac{\sqrt{2 z}}{1-\beta}\right) .
$$

Иначе говоря, при $N \rightarrow \infty$ виполняется

$$
N\left(\frac{q_{N}(0) q_{N+1}\left(c N^{2 \beta-2} z\right)}{q_{N+1}(0) q_{N}\left(c N^{2 \beta-2} z\right)}-1\right) \rightarrow \sqrt{2 z} J_{\frac{\alpha}{1-\beta}+1}\left(\frac{\sqrt{2 z}}{1-\beta}\right) / J_{\frac{\alpha}{1-\beta}}\left(\frac{\sqrt{2 z}}{1-\beta}\right)
$$

сходимость в сферической метрике равномерная на компактах $K \subset \mathbb{C}$. 
ЗАмЕчАниЕ 1. При доказательстве теоремы 1 функция $f(z)$ сперва получается в неупрошенном виде и определяется через семейство решений краевых задач для дифференциальных уравнений Риккати. Для каждого фиксированного $z$ построим функцию $h_{z}(t)$ :

$$
h_{z}^{\prime}(t)=2 z t^{1-2 \beta}-\frac{2 \alpha h_{z}(t)}{t}+\frac{h_{z}^{2}(t)}{t}, \quad \varlimsup_{t \rightarrow+0} t^{2 \beta-2}\left|h_{z}(t)\right|<\infty .
$$

Тогда $f(z)=h_{z}(1)$. Таким образом, в данном случае значения $f(z)$ представимы в терминах функций Бесселя. Однако общая форма описания асимптотики отношения через решения уравнений Риккати должна сохраниться и при других видах асимптотики $b_{n}$ или асимптотики величины из формулы (5). При этом изменится вид зависимости коэффициентов уравнений Риккати от $t$ и решения уравнений Риккати уже не обязательно будут выражаться через функции Бесселя.

СлЕДСТВИЕ. В условиях теоремь 1 последовательность полиномов $\left\{q_{n}(x)\right\}$ имеет следующую асимптотику нулей:

$$
x_{k, n} \sim \frac{c(1-\beta)^{2} j_{k}^{2}\left(\frac{\alpha}{1-\beta}\right)}{2} n^{2 \beta-2}
$$

где через $j_{k}(s)$ обозначен $k$-й положстельньй корень функиии Бесселя $J_{s}(x)$.

ЗАмЕчАниЕ 2. Отметим, что условия теоремы 1 (ограничения на поведение коэффициентов рекуррентных соотношений) более общие, чем в теореме А, которой соответствует случай $\beta=0$. И хотя результат в теореме А выглядит более точньг (в теореме А найдена асимптотика полиномов, а в теореме 1 асимптотика отношений последовательных полиномов), но даже в условиях теоремы $\mathrm{A}(\beta=0)$ формула $\left(7^{\prime}\right)$ непосредственно не следует из (3), а может быть получена только при наложении дополнительных условий на остаток в (3).

ЗАмЕчАниЕ 3. Каждое из ограничений на параметры в (6) имеет естественньй для поставленной задачи характер. А именно, первое неравенство в (6) гарантирует определенность проблемы моментов, противоположное неравенство означает, что проблема моментов неопределена, а в случае равенства все зависит от последующих членов асимптотики. Такая же связь есть между вторым неравенством в (6) и условием "в точке 0 отсутствует сосредоточенная масса".

Область приложений теоремы 1 - асимптотика нулей ортогональных многочленов. Например, можно получить асимптотику крайних нулей для полиномов Якоби и их “шевелений” (случай $\beta=0$ ), а также асимптотику в окрестности точки $x_{0}=0$ нулей полиномов Лагерра и их “шевелений” (случай $\beta=\frac{1}{2}$ ). Кроме того, в работе [2] была установлена связь точных констант в неравенствах Бернштейна-Маркова для некоторых весовых пространств с первыми нулями полиномов, удовлетворяющих трехчленным рекуррентньм соотношениям. Эти полиномы соответствуют случаю $\beta=-1$.

Условия теоремы 1 содержат как исходные данные асимптотику значений многочленов в точке и асимптотику рекуррентных коэффициентов. Эти два объекта, очевидно, связаны между собой. Однако знание одной лишь асимптотики рекуррентных коэффициентов с какой угодно точностью недостаточно для анализа поведения значений многочленов в точке (пример: многочлены Чебышёва первого и 
второго рода при $\left.x_{0}=1\right)$. Возникает вопрос об определении необходимых условий на рекуррентные коэффициенты, при которых возможно выполнение условий теоремы 1 при некоторых значениях параметров $\alpha, \beta$. Частичньй ответ на этот вопрос дает теорема 2 настоящей работы.

Теорема 2. Для того чтобы последовательность многочленов $\left\{q_{n}\right\}$, определенная соотношениями (4), имела значения $q_{n}(0)$, удовлетворяющие условию (5), необходимо выполнение следующего условия на рекуррентные коэффичиентьи:

$$
\sum_{k=n}^{\infty}\left(\frac{b_{k-1} b_{k}}{\Delta_{k}}-4\right)=\frac{4 \alpha^{2}-1}{n}+o\left(\frac{1}{n}\right)
$$

Мы видим, что величина $\alpha^{2}$ определяется только асимптотикой рекуррентных коэффициентов, но знак $\alpha$ неизвестен. По-видимому, условие (8) является также и достаточным в таком смысле: если (8) выполнено, то оно задает два возможных значения $\alpha$ и для какого-то из них верно соотношение (5); при подходящем изменении конечного числа первых рекуррентных коэффициентов соотношение (5) будет верно для любого заданного из двух $\alpha$. Однако это предположение требует дополнительного исследования. Кроме того, здесь возникает вопрос о поведении полиномов для рекуррентных коэффициентов, удовлетворяющих формуле (8) при чисто мнимых $\alpha$.

Статья разделена на 6 частей. Параграфы 2-4 посвящены доказательству теорем 1 и 2. В $\S 5$ приводятся примеры приложения результатов к асимптотике классических ортогональных многочленов и асимптотике точных констант в неравенствах Маркова-Бернштейна. Последний $\S 6$ посвящен обсуждению возможных расширений методов, представленных в статье.

\section{§ 2. Принцип доказательства и вспомогательные леммы}

Перейдем к доказательству теоремы 1, попутно формулируя и доказывая необходимые леммы. Вначале будет изложен общий подход, а затем мы продолжим доказательство, используя конкретньй вид асимптотики начальных данных.

Возьмем некоторое $x$. Определим последовательности $\left\{r_{n}\right\}$ и $\left\{F_{n}\right\}$ следующими соотношениями:

$$
-\frac{b_{n} q_{n}(0)}{q_{n+1}(0)}=r_{n}+1, \quad \frac{q_{n}(x)}{q_{n-1}(x)}=\frac{q_{n}(0)}{q_{n-1}(0)}\left(1-\frac{F_{n}}{n}\right) .
$$

Последовательность $\left\{r_{n}\right\}$ определяется только рекуррентными коэффициентами. Последовательность $\left\{F_{n}\right\}$ зависит также и от взятого $x$. Подставив в (4) значение $x=0$, можно выразить $\Delta_{n}$. Теперь подставим это $\Delta_{n}$ и произведем сокрашения. Получим:

$$
1-\frac{F_{n+1}}{n+1}=\left(1-\frac{x}{b_{n}}\right)\left(1+r_{n}\right)-\frac{n r_{n}}{n-F_{n}} \quad(n>0), \quad F_{1}=\frac{x}{b_{0}}
$$

или

$$
F_{n+1}=(n+1)\left(\frac{x}{b_{n}}\left(1+r_{n}\right)+\frac{r_{n} F_{n}}{n-F_{n}}\right) \quad(n>0), \quad F_{1}=\frac{x}{b_{0}}
$$


Видно, что $F_{n+1}$ получается из $F_{n}$ действием дробно-линейного преобразования. Основная идея нахождения аппроксимации для $F_{n}$ состоит в подборе инфинитезимального преобразования ( "поля направлений”), у которого действие сдвига на 1 есть дробно-линейное преобразование, близкое к выписанному. Именно поэтому мы рассматриваем уравнения Риккати, так как у них операторы сдвига - дробно-линейные преобразования. Подробнее связь уравнений Риккати и дробно-линейных преобразований раскрыта в следующей лемме.

Лемма 1. 1) Для любого решения уравнения Риккати $y^{\prime}=p(t)+2 q(t) y+$ $r(t) y^{2}$ с непрерывными коэффичиентами $p, q, r$ справедлива формула сдвига аргумента:

$$
y(t)=\frac{A(s, t) y(s)+B(s, t)}{C(s, t) y(s)+D(s, t)} .
$$

При этом функции $A, B, C, D$ не зависят от взятого решения и находятся из условий:

$$
\begin{gathered}
\frac{d}{d t}\left(\begin{array}{cc}
A(s, t) & B(s, t) \\
C(s, t) & D(s, t)
\end{array}\right)=\left(\begin{array}{cc}
q(t) & p(t) \\
-r(t) & -q(t)
\end{array}\right)\left(\begin{array}{ll}
A(s, t) & B(s, t) \\
C(s, t) & D(s, t)
\end{array}\right) \\
\left(\begin{array}{ll}
A(s, s) & B(s, s) \\
C(s, s) & D(s, s)
\end{array}\right)=\left(\begin{array}{ll}
1 & 0 \\
0 & 1
\end{array}\right)
\end{gathered}
$$

Нормировка при таком определении будет $A D-B C \equiv 1$.

2) Любое решение матричного уравнения

$$
\frac{d}{d t} M=\left(\begin{array}{cc}
q(t) & p(t) \\
-r(t) & -q(t)
\end{array}\right) M
$$

обладает свойством $\operatorname{det}(M)=$ const. Eсли $\operatorname{det}(M) \neq 0$, то матрица из $n .1)$ леммы (т.е. решение задачи (12)) представима в виде

$$
\left(\begin{array}{ll}
A(s, t) & B(s, t) \\
C(s, t) & D(s, t)
\end{array}\right)=M(t)[M(s)]^{-1} .
$$

3) Возъмем любое решение уравнения (13) с не равным нулю определителем (определитель - константа). Тогда общее решение уравнения Риккати $y^{\prime}(t)=p(t)+2 q(t) y(t)+r(t) y^{2}(t)$ мохет быть записано в виде

$$
y(t)=\frac{M_{11}(t)+\lambda M_{12}(t)}{M_{21}(t)+\lambda M_{22}(t)}, \quad \lambda=\mathrm{const} .
$$

Для построения решения уравнения (13) с таким свойством достаточно взять два линейно независимых векторных решения такого жсе уравнения в качестве столбиов. 
ДокАЗАТЕльство. Начнем с п. 3) леммы. Для этого просто подставим правую часть соотношения (14) в дифференциальное уравнение. Используем соотношение (13), расписанное по компонентам, для избавления от производных. Получим

$$
\begin{aligned}
& \frac{d}{d t}\left(\frac{M_{11}(t)+\lambda M_{12}(t)}{M_{21}(t)+\lambda M_{22}(t)}\right)=\frac{M_{11}^{\prime}+\lambda M_{12}^{\prime}}{M_{21}+\lambda M_{22}}-\frac{\left(M_{11}+\lambda M_{12}\right)\left(M_{21}^{\prime}+\lambda M_{22}^{\prime}\right)}{\left(M_{21}+\lambda M_{22}\right)^{2}} \\
& \quad=q(t)\left(\frac{M_{11}+\lambda M_{12}}{M_{21}+\lambda M_{22}}\right)+p(t)+\left(\frac{M_{11}+\lambda M_{12}}{M_{21}+\lambda M_{22}}\right)\left(r(t) \frac{M_{11}+\lambda M_{12}}{M_{21}+\lambda M_{22}}+q(t)\right) \\
& \quad=p(t)+2 q(t) \frac{M_{11}(t)+\lambda M_{12}(t)}{M_{21}(t)+\lambda M_{22}(t)}+r(t)\left(\frac{M_{11}(t)+\lambda M_{12}(t)}{M_{21}(t)+\lambda M_{22}(t)}\right)^{2} .
\end{aligned}
$$

Итак, получили тождество. Значит, формула (14) действительно задает решение уравнения Риккати при произвольном $\lambda$. Так как $\operatorname{det}(M) \neq 0$, то (14) есть формула для обшего решения. Далее, опять из покомпонентной записи (13) после подстановок и упрошений получим тождество $(\operatorname{det}(M(t)))^{\prime}=0$. Непосредственно из (13) следует, что столбцы $M$ изменяются независимо. Поэтому, во-первых, матрицу $M$ действительно можно получить из векторов (что завершает доказательство п. 3)), а во-вторых, умножение решения (13) на постоянную матрицу справа вновь даст решение (13). По теореме единственности отсюда следует п. 2) леммы. Наконец, из пाп. 2) и 3) отображение

$$
f: t \rightarrow \frac{A(s, t) y(s)+B(s, t)}{C(s, t) y(s)+D(s, t)}
$$

будет решением уравнения Риккати, упоминаемого в п. 1) леммы 1 . Подстановка $t=s$ дает $f(s)=y(s)$, поэтому $f$ и $y$ совпадают по теореме единственности задачи Коши для дифференциального уравнения. Это совпадение пока что доказано только локально, до ближайшего полюса, но после применения отображения $y \rightarrow 1 / y$ аналогичные рассуждения покажут, что совпадение сохраняется при переходе через полюс решения. Мы также можем убедиться, что подстановка $t=s$ дает условие нормировки.

Итак, мы можем рассматривать дробно-линейные операторы сдвига для уравнений Риккати. Для обеспечения близости этих преобразований с преобразованиями (10) нужно уметь сравнивать результаты близких преобразований в близких точках. Таким метрическим свойствам дробно-линейных функций посвящены две нижеследующие леммы: в первой оценивается результат действия одного и того же преобразования на две разные точки, а во второй - разных преобразований на одну и ту же точку.

ОПРЕДЕЛЕниЕ. Сферическим расстоянием между двумя точками $z, w \in \overline{\mathbb{C}}_{\text {на- }}$ зывается величина

$$
\rho(z, w)=\frac{|z-w|}{\sqrt{\left(1+|z|^{2}\right)\left(1+|w|^{2}\right)}}, \quad \rho(z, \infty)=\lim _{w \rightarrow \infty} \rho(z, w) .
$$

Это стандартное определение, и хорошо известно, что $\rho$ - действительно метрика для $\overline{\mathbb{C}}$ со свойствами:

$$
\forall z, w \in \overline{\mathbb{C}} \quad \rho(z, w) \leqslant 1, \quad \forall z \in \overline{\mathbb{C}} \quad \rho\left(z, \frac{1}{\bar{z}}\right)=1 .
$$


ЛЕмма 2. Пусть $\varphi(t)=(a t+b) /(c t+d)-$ любая дробно-линейная функиия. Обозначим через $T$ и $\Delta$ такие выражсения от коэффичиентов:

$$
T=|a|^{2}+|b|^{2}+|c|^{2}+|d|^{2}, \quad \Delta=|a d-b c| \quad(\neq 0) .
$$

Тогда верно следующее соотношение:

$$
\forall z, w \in \overline{\mathbb{C}} \quad \rho(\varphi(z), \varphi(w)) \leqslant\left(\frac{T}{2 \Delta}+\sqrt{\left(\frac{T}{2 \Delta}\right)^{2}-1}\right) \rho(z, w) .
$$

Эта величина может быть также записана в таком виде:

$$
\text { Пусть } \quad M=\left(\begin{array}{ll}
a & b \\
c & d
\end{array}\right) . \quad \text { Тогда } \frac{\rho(\varphi(z), \varphi(w))}{\rho(z, w)} \leqslant \frac{\|M\|^{2}}{|\operatorname{det}(M)|} .
$$

ДоКАЗАТЕЛьство. Преобразуем и оценим супремум отношения:

$$
\begin{aligned}
\sup _{z, w \in \overline{\mathbb{C}}} \frac{\rho(\varphi(z), \varphi(w))}{\rho(z, w)} & =\sup _{z, w \in \overline{\mathbb{C}}} \frac{|a d-b c| \sqrt{(1+z \bar{z})(1+w \bar{w})}}{\sqrt{\left(|a z+b|^{2}+|c z+d|^{2}\right)\left(|a w+b|^{2}+|c w+d|^{2}\right)}} \\
& \leqslant \sup _{z \in \overline{\mathbb{C}}} \frac{|a d-b c|(1+z \bar{z})}{(a \bar{a}+c \bar{c}) z \bar{z}+2 \operatorname{Re}((a \bar{b}+c \bar{d}) z)+(b \bar{b}+d \bar{d})} \\
& \leqslant \sup _{z \in \overline{\mathbb{C}}} \frac{|a d-b c|\left(1+|z|^{2}\right)}{(a \bar{a}+c \bar{c})|z|^{2}-2|a \bar{b}+c \bar{d}||z|+(b \bar{b}+d \bar{d})} \\
& =\left(\frac{T}{2 \Delta}+\sqrt{\left(\frac{T}{2 \Delta}\right)^{2}-1}\right) .
\end{aligned}
$$

Анализируя неравенства, можно показать, что на самом деле здесь сквозное равенство, но супремум не достигается. Далее, величины $T$ и $\Delta$ можно записать так: $T=\operatorname{Tr}\left(M M^{*}\right), \Delta=|\operatorname{det}(M)|=\sqrt{\operatorname{det}\left(M M^{*}\right)}$. Это доказывает вторую часть леммы.

ЛЕмма 3. Пусть $\varphi(t)=\left(a_{1} t+b_{1}\right) /\left(c_{1} t+d_{1}\right) u \psi(t)=\left(a_{2} t+b_{2}\right) /\left(c_{2} t+d_{2}\right)$ - любъе две дробно-линейные функиии, $a_{i} d_{i}-b_{i} c_{i} \neq 0$ для $i=1,2$. Тогда справедлив следующий результат:

$$
\sup _{z \in \overline{\mathbb{C}}} \rho(\varphi(z), \psi(z))= \begin{cases}1 & \text { nри } S>R, \\ \frac{\sqrt{(R+P)(S+P)}+\sqrt{(R-P)(S-P)}}{R+S} & \text { иначе }\end{cases}
$$

әде величинь $P, R, S$ можно вычислить по таким выражсениям:

$$
\begin{aligned}
& P=\left|\left(\left(a_{1} d_{2}-a_{2} d_{1}\right)+\left(c_{1} b_{2}-c_{2} b_{1}\right)\right)^{2}+4\left(b_{1} a_{2}-b_{2} a_{1}\right)\left(c_{1} d_{2}-c_{2} d_{1}\right)\right| \\
& R=\left|\left(a_{1} d_{2}+a_{2} d_{1}\right)-\left(c_{1} b_{2}+c_{2} b_{1}\right)\right|^{2}+4\left|\left(a_{1} d_{1}-b_{1} c_{1}\right)\left(a_{2} d_{2}-b_{2} c_{2}\right)\right| \\
& S=\left|\left(a_{1} d_{2}-a_{2} d_{1}\right)+\left(c_{1} b_{2}-c_{2} b_{1}\right)\right|^{2}+2\left|b_{1} a_{2}-b_{2} a_{1}\right|^{2}+2\left|c_{1} d_{2}-c_{2} d_{1}\right|^{2}
\end{aligned}
$$


СлЕДСТВИЕ. Имеет место соотношение

$\rho(\varphi(z), \psi(z)) \leqslant \sqrt{\frac{\left|a_{1} d_{2}-a_{2} d_{1}\right|^{2}+\left|c_{1} b_{2}-c_{2} b_{1}\right|^{2}+\left|b_{1} a_{2}-b_{2} a_{1}\right|^{2}+\left|c_{1} d_{2}-c_{2} d_{1}\right|^{2}}{\left|a_{1} d_{1}-b_{1} c_{1}\right|\left|a_{2} d_{2}-b_{2} c_{2}\right|}}$.

ДоКАЗАТЕЛЬСТВО ЛЕМмЫ 3 и СЛЕДСТВИЯ. Прежде всего отметим, что при сделанных предположениях на параметры $\varphi$ и $\psi$ выражение под супремумом непрерывно по параметрам, а значит, значение супремума тоже непрерывно по параметрам (относительно множества, на котором супремум достигается, этого сказать нельзя, но нас оно не интересует). Поэтому достаточно найти супремум в предположении, что параметры находятся в общем положении, и продолжить формулу по непрерывности. Далее, это выражение имеет два легко проверяемых свойства инвариантности. Пусть $h(t)=\left(a_{3} t+b_{3}\right) /\left(c_{3} t+d_{3}\right)$. Тогда имеем

$$
\begin{array}{ll}
\sup _{z \in \overline{\mathbb{C}}} \rho(\varphi(z), \psi(z))=\sup _{z \in \overline{\mathbb{C}}} \rho(\varphi(h(z)), \psi(h(z))) \text { при } & \left(\begin{array}{ll}
a_{3} & b_{3} \\
c_{3} & d_{3}
\end{array}\right) \in G L(2, \mathbb{C}), \\
\sup _{z \in \overline{\mathbb{C}}} \rho(\varphi(z), \psi(z))=\sup _{z \in \overline{\mathbb{C}}} \rho(h(\varphi(z)), h(\psi(z))) \text { при } & \left(\begin{array}{ll}
a_{3} & b_{3} \\
c_{3} & d_{3}
\end{array}\right) \in S U(2, \mathbb{C}) .
\end{array}
$$

Пользуясь первым свойством, можно свести задачу к случаю, когда $\psi$ равно тождественному преобразованию. При этом $\varphi$ перейдет в $\varphi \circ \psi^{-1}$, про которое тоже можно сказать, что его параметры находятся в общем положении. Тогда оно имеет две неподвижных точки $z_{1}$ и $z_{2}$, и пусть $z_{1} \bar{z}_{2} \neq-1$. В этом случае можно, пользуясь вторым свойством, перевести их в точки вида $\pm t, t \in(0 ; 1)$.

Общий вид дробно-линейного преобразования, имеющего неподвижные точки $t$ и $-t$, есть $f(z)=\left(k z+t^{2}\right) /(z+k)$. Для получения ответа в приведенной таким образом задаче сделаем дальнейшие преобразования с целью получить упрошение за счет симметрии. Пусть $k=t\left(s+s^{-1}\right) / 2$, где $|s|<1$. Сделаем замену $z=$ $t(s y-1) /(s-y)$. Тогда $f(z)=t(s y+1) /(s+y)$. Получаем

$$
\begin{aligned}
\rho(f(z), z) & =\rho\left(t \frac{s y+1}{s+y}, t \frac{s y-1}{s-y}\right) \\
& =\frac{2 t\left|s\left(y^{2}-1\right)\right|}{\sqrt{\left(|s+y|^{2}+t^{2}|s y+1|^{2}\right)\left(|s-y|^{2}+t^{2}|s y-1|^{2}\right)}} .
\end{aligned}
$$

Теперь рассмотрим отдельно два случая: $|s|<t$ и $|s|>t$. В первом случае покажем, что подстановка значения $y=0$ дает максимум рассматриваемого выражения. Имеем

$$
\begin{aligned}
& \frac{1}{\rho^{2}(f(z), z)}-\frac{1}{\rho^{2}(t / s,-t / s)} \\
& \quad=\frac{\left(1-t^{4}\right)\left(1-|s|^{4}\right)|y|^{4}+2\left|1-s^{2}\right|^{2}|y|^{2}\left(t^{2}+\operatorname{Re}\left(\frac{s^{2}-t^{4}}{1-s^{2}} \frac{\bar{y}}{y}\right)\right)}{4 t^{2}|s|^{2}\left|1-y^{2}\right|^{2}} .
\end{aligned}
$$

При $|s|<t$ имеет место $\left|s^{2}-t^{4}\right|<t^{2}\left|1-s^{2}\right|$, поэтому числитель и знаменатель всегда положительны. Во втором случае можно выбрать такое значение $y$, при 
котором расстояние равно единице - это, очевидно, будет максимум. Возможные значения $y$ такие:

$$
y= \pm \sqrt{\left(\frac{s-t^{2} \bar{s}}{\bar{s}-t^{2} s}\right)\left(\frac{s \bar{s}-t^{2}}{1-t^{2} s \bar{s}}\right)}
$$

Таким образом, получен ответ в приведенной задаче. Теперь осталось написать его в терминах коэффициентов $\varphi$ и $\psi$. Как мы видели, после использования первого из свойств (15) мы приходим к функции $\varphi \circ \psi^{-1}$, матрица коэффициентов которой будет такой:

$$
\left(\begin{array}{ll}
a & b \\
c & d
\end{array}\right)=\left(\begin{array}{ll}
a_{1} & b_{1} \\
c_{1} & d_{1}
\end{array}\right)\left(\begin{array}{ll}
a_{2} & b_{2} \\
c_{2} & d_{2}
\end{array}\right)^{-1}=\left(\begin{array}{ll}
a_{1} d_{2}-b_{1} c_{2} & b_{1} a_{2}-a_{1} b_{2} \\
c_{1} d_{2}-d_{1} c_{2} & d_{1} a_{2}-c_{1} b_{2}
\end{array}\right)
$$

Для преобразования на основе второго из свойств (15) мы не будем выписывать результат как матрицу. Вместо этого выразим значение супремума через инварианты. Их можно найти, например, используя методы нахождения инвариантов действия групп Ли. В данном случае с учетом тождественных преобразований вида “умножение числителя и знаменателя на константу” получим, что есть один комплексньй и один вещественный инварианты:

$$
I_{1}=\frac{(a+d)^{2}}{a d-b c}, \quad I_{2}=\frac{|a|^{2}+|b|^{2}+|c|^{2}+|d|^{2}}{|a d-b c|}
$$

Значения инвариантов для функции $f(z)$ из приведенной задачи будут

$$
I_{1}=4\left(\frac{s^{2}+1}{s^{2}-1}\right)^{2}, \quad I_{2}=2\left|\frac{s^{2}+1}{s^{2}-1}\right|^{2}+\frac{4|s|^{2}\left(1+t^{4}\right)}{t^{2}\left|s^{2}-1\right|^{2}} .
$$

Рассмотрим следующие выражения от коэффициентов:

$$
R=|a+d|^{2}+4|a d-b c|, \quad S=|a-d|^{2}+2|b|^{2}+2|c|^{2}, \quad P=\left|(a-d)^{2}+4 b c\right|
$$

и выразим их отношения через значения инвариантов:

$$
\frac{R}{P}=\frac{\left|I_{1}\right|+4}{\left|I_{1}-4\right|}=\frac{1}{2}\left(|s|^{-2}+|s|^{2}\right), \quad \frac{S}{P}=\frac{2 I_{2}-\left|I_{1}\right|}{\left|I_{1}-4\right|}=\frac{1}{2}\left(t^{-2}+t^{2}\right) .
$$

В соответствии с выбором переменных: $t \in(0,1)$ и $|s|<1$ получаем, что $|s|<t \Longleftrightarrow$ $R>S$. Теперь выражаем sup $\rho$ (первый случай):

$$
\begin{aligned}
\rho\left(\frac{t}{s}, \frac{-t}{s}\right) & =\frac{2 t|s|}{|s|^{2}+t^{2}}=\frac{4}{\left(|s|^{-1}+|s|\right)\left(t^{-1}+t\right)-\left(|s|^{-1}-|s|\right)\left(t^{-1}-t\right)} \\
& =\frac{2 P}{\sqrt{(R+P)(S+P)}-\sqrt{(R-P)(S-P)}} \\
& =\frac{\sqrt{(R+P)(S+P)}+\sqrt{(R-P)(S-P)}}{R+S} .
\end{aligned}
$$


Подстановка выражений для $a, b, c, d$ из (16) завершает доказательство леммы. Для получения следствия осталось провести несколько оценок:

$$
\frac{\sqrt{(R+P)(S+P)}+\sqrt{(R-P)(S-P)}}{R+S} \leqslant \frac{\sqrt{S+P}+\sqrt{S-P}}{\sqrt{R+S}} \leqslant \frac{2 \sqrt{S}}{\sqrt{R+S}} .
$$

Последнюю величину можно использовать и в случае $S>R$. Для окончания доказательства оценим отдельно числитель и знаменатель:

$$
\begin{aligned}
S & \leqslant S+\left|\left(a_{1} d_{2}-a_{2} d_{1}\right)-\left(c_{1} b_{2}-c_{2} b_{1}\right)\right|^{2} \\
& =2\left(\left|a_{1} d_{2}-a_{2} d_{1}\right|^{2}+\left|c_{1} b_{2}-c_{2} b_{1}\right|^{2}+\left|b_{1} a_{2}-b_{2} a_{1}\right|^{2}+\left|c_{1} d_{2}-c_{2} d_{1}\right|^{2}\right), \\
S+R & =2\left(|a|^{2}+|b|^{2}+|c|^{2}+|d|^{2}\right)+4|a d-b c| \\
& \geqslant 8|a d-b c|=8\left|a_{1} d_{1}-b_{1} c_{1}\right|\left|a_{2} d_{2}-b_{2} c_{2}\right| .
\end{aligned}
$$

Таким образом, мы получили возможность оценивать величину ошибки, возникающей при замене дробно-линейных преобразований (10) на близкие. Но использование асимптотической информации дает эффект лишп при достаточно больших $n$. Поэтому в ситуации, когда $x$ близок к 0 , а $n$ мало (в каком-то смысле), величины $F_{n}$ будут оцениваться непосредственно. Из соображений непрерывности $F_{n}$ должны быть в окрестности нуля, так как формула $(10)$ показывает, что при $x=0$ получится $F_{1}=0$, и из $F_{n}=0$ следует $F_{n+1}=0$.

Перейдем к изложению данной схемы с учетом конкретных асимптотических данных.

\section{§3. Окончание доказательства теоремы 1}

Фиксируем компакт $K$ в $\mathbb{C}$. Теперь выберем $z \in K$ и $N$ и рассмотрим формулу (10) при $x$, равном $c N^{2 \beta-2} z$. Докажем, что для номеров $n$, достаточно малых по сравнению с $N$, можно непосредственно оценить порядок $F_{n}$. Сформулируем лемму.

ЛЕМма 4. Существуют положительные постоянные (зависящие только от выбранного компакта $K) k, p, \varepsilon$ такие, что всегда

$$
\left|F_{n}\right|<k\left(\frac{n+1}{n}\right)^{-p}\left(\frac{n}{N}\right)^{2-2 \beta} \quad n p u \quad n-1<\varepsilon N .
$$

ДокАЗАТЕЛЬСТво. Построим нужные константы и затем индукцией докажем утверждение леммы. Пусть $C_{1}$ - следующая величина:

$$
C_{1}=c \cdot \max _{z \in K}|z| \cdot \max _{n \in \mathbb{N}}\left(\frac{n^{2 \beta}\left(1+r_{n}\right)}{b_{n}}\right) .
$$

Обозначим $2(1+\alpha-\beta) / 3$ через $\delta$. Из условия (6) $\delta>0$. Из условия (5) и определения $r_{n}$ получаем, что

$$
\frac{(n+1) r_{n}}{n-\delta}=1+\frac{\delta-2 \alpha}{n}+o\left(\frac{1}{n}\right)
$$


поэтому можно выбрать $p$ так, что при всех $n \in \mathbb{N}$

$$
\left(1+\frac{1}{n(n+2)}\right)^{-p} \frac{(n+1) r_{n}}{n-\delta}<1+\frac{2 \delta-2 \alpha}{n+1} .
$$

Теперь последовательно определим нужные $k$ и $\varepsilon$ из условий:

$$
4\left(\frac{3}{2}\right)^{p} \frac{C_{1}}{k}<\delta, \quad 2^{p} \frac{c \max |z|}{b_{0} k}<1, \quad k \varepsilon^{2-2 \beta}<\delta .
$$

Тогда при $n=1$ нужная оценка верна для всех $N$. Предположим, что при некотором $n$ мы знаем, что оценка (17) выполнена и что $n<\varepsilon N$. Оценим $\left|F_{n+1}\right|$ из (10), пользуясь (18)-(20):

$$
\begin{aligned}
& \left|F_{n+1}\right|<(n+1)\left[C_{1} N^{2 \beta-2} n^{-2 \beta}+k\left(\frac{n+1}{n}\right)^{-p} N^{2 \beta-2} n^{2-2 \beta} \frac{r_{n}}{n-k \varepsilon^{2-2 \beta}}\right] \\
& <k\left(\frac{N}{n}\right)^{2 \beta-2}\left(\frac{n+2}{n+1}\right)^{-p}\left[\frac{n+1}{n^{2}}\left(\frac{n+2}{n+1}\right)^{p} \frac{C_{1}}{k}+\left(1+\frac{1}{n(n+2)}\right)^{-p} \frac{(n+1) r_{n}}{n-\delta}\right] \\
& <k\left(\frac{N}{n}\right)^{2 \beta-2}\left(\frac{n+2}{n+1}\right)^{-p}\left[\frac{\delta}{n+1}+1+\frac{2 \delta-2 \alpha}{n+1}\right]<k\left(\frac{n+2}{n+1}\right)^{-p}\left(\frac{N}{n+1}\right)^{2 \beta-2} .
\end{aligned}
$$

Индуктивный переход для формулы (17) сделан, лемма доказана.

Теперь изучим поведение $F_{n}$ для номеров $n$ того же порядка, что и $N$, сравнивая последовательность $\left\{F_{n}\right\}$ с решением уравнения Риккати. Возьмем некоторое $\varepsilon_{1}$, $0<\varepsilon_{1}<\varepsilon$. Обозначим $N_{1}=\left[N \varepsilon_{1}\right]+1$, построим решение $y_{1}$ задачи Коши

$$
y^{\prime}(t)=2 z t^{1-2 \beta}-\frac{2 \alpha y(t)}{t}+\frac{y^{2}(t)}{t}, \quad y\left(\frac{N_{1}}{N}\right)=F_{N_{1}}
$$

и оценим величины $\rho\left(F_{n}, y_{1}(n / N)\right)$ при $N_{1} \leqslant n \leqslant N$, пользуясь леммами 1-3. Имеем: при $n=N_{1}$ расстояние равно 0; из формулы (10) переход от $F_{n}$ к $F_{n+1}$ осуществляется дробно-линейньм преобразованием с матрицей коэффициентов

$$
\begin{aligned}
M_{1} & =\left(\begin{array}{cc}
\frac{(n+1)\left[r_{n}-\left(1+r_{n}\right) c z N^{2 \beta-2}\right]}{b_{n}} & \frac{\left(1+r_{n}\right) n(n+1) c z N^{2 \beta-2}}{b_{n}} \\
-1 & n
\end{array}\right) \\
& =\left(\begin{array}{cc}
n-2 \alpha+o(1) & 2 z\left(\frac{n}{N}\right)^{2-\beta}(1+o(1)) \\
-1 & n
\end{array}\right), \quad \operatorname{det}\left(M_{1}\right)=n(n+1) r_{n}
\end{aligned}
$$

а для перехода от $y_{1}(n / N)$ к $y_{1}(n+1 / N)$ матрицу коэффициентов можно найти с достаточной для наших целей точностью, если подставить в формулы $(11),(12)$ коэффициенты из уравнения задачи Кошш (21), взять $s=n / N$ и написать ряд Тейлора для точки $t=(n+1) / N$. Получится матрица

$$
\begin{aligned}
M_{2} & =E+\frac{1}{N}\left(\begin{array}{cc}
-\frac{\alpha}{s} & 2 z s^{1-2 \beta} \\
-\frac{1}{s} & \frac{\alpha}{s}
\end{array}\right)+\frac{1}{N^{2}}\left(\begin{array}{cc}
O\left(s^{-2}\right) & O\left(z s^{-2 \beta}\right) \\
O\left(s^{-2}\right) & O\left(s^{-2}\right)
\end{array}\right) \\
& =\left(\begin{array}{cc}
1-\frac{\alpha}{n}+O\left(n^{-2}\right) & 2 z N^{2 \beta-2}\left(n^{1-2 \beta}+O\left(n^{-2 \beta}\right)\right) \\
-\frac{1}{n}+O\left(n^{-2}\right) & 1+\frac{\alpha}{n}+O\left(n^{-2}\right)
\end{array}\right) .
\end{aligned}
$$


Обозначим через $\tilde{y}$ решение уравнения (21) с другим начальным условием:

$$
\tilde{y}(n / N)=F_{n} .
$$

Тогда из леммы 2 можно оценить $\rho(y(n+1 / N), \tilde{y}(n+1 / N))$, а из леммы 3 - оценить $\rho\left(\tilde{y}(n+1 / N), F_{n+1}\right)$. Сушествует константа $C$ такая, что $\left\|M_{2}\right\|<[(n+1) / n]^{C}$, причем $C>0$. Получаем:

$$
\begin{aligned}
\rho\left(y_{1}\left(n+\frac{1}{N}\right), F_{n+1}\right) & \leqslant \rho\left(y_{1}\left(n+\frac{1}{N}\right), \tilde{y}\left(n+\frac{1}{N}\right)\right)+\rho\left(\tilde{y}\left(n+\frac{1}{N}\right), F_{n+1}\right) \\
& <\left\|M_{2}\right\| \rho\left(y_{1}\left(\frac{n}{N}\right), \tilde{y}\left(\frac{n}{N}\right)\right)+\sqrt{\frac{o(1)}{n(n+1)}} r_{n} \\
& <\left(\frac{n+1}{n}\right)^{C} \rho\left(y_{1}\left(\frac{n}{N}\right), F_{n}\right)+o\left(n^{-1}\right) .
\end{aligned}
$$

Отсюда следует, что

$$
(n+1)^{-C} \rho\left(y_{1}\left(n+\frac{1}{N}\right), F_{n+1}\right)<n^{-C} \rho\left(y_{1}\left(\frac{n}{N}\right), F_{n}\right)+o\left(n^{-1-C}\right) .
$$

Учитывая, что $y\left(N_{1} / N\right)=F_{N_{1}}$, получаем оценку: при $N_{1} \leqslant n \leqslant N$

$$
\rho\left(y_{1}\left(\frac{n}{N}\right), F_{n}\right)<n^{C} \sum_{k=N_{1}}^{n-1} o\left(k^{-1-C}\right)<\frac{\varepsilon_{1}^{-C}-1}{C} o(1) .
$$

Итак, при фиксированном $\varepsilon_{1}$ и $N \rightarrow \infty$ мы получаем, что $\rho\left(y_{1}(n / N), F_{n}\right) \rightarrow 0$ для $n \in\left[N_{1}, N\right]$ равномерно по $n$. Поэтому осталось показать сходимость решений задач Коши (21). Для этого проведем следующие рассуждения. Заменим $\varepsilon_{1}$ на $\varepsilon_{2}$ и построим решения $y_{2}$ аналогично $y_{1}$ из $(21)$. Тогда имеем: при $N \rightarrow \infty$ и любом $n \in\left[\max \left(N_{1}, N_{2}\right), N\right]$

$$
\rho\left(y_{2}\left(\frac{n}{N}\right), y_{1}\left(\frac{n}{N}\right)\right)<\sup _{n \in\left[N_{1}, N\right]} \rho\left(y_{1}\left(\frac{n}{N}\right), F_{n}\right)+\sup _{n \in\left[N_{2}, N\right]} \rho\left(F_{n}, y_{2}\left(\frac{n}{N}\right)\right) \rightarrow 0 .
$$

Этот вывод годится для любого $0<\varepsilon_{2}<\varepsilon_{1}$, так что теперь можно устремить $\varepsilon_{2} \mathrm{\kappa}$ нулю и воспользоваться леммами 1 и 4 . Применим лемму 1 к дифференциальному уравнению из (21). Соответствующее ему уравнение (13) имеет линейно независимые решения-столбцы с таким поведением: первый - основной для нашей задачи

$$
\begin{gathered}
\Gamma\left(1+\frac{\alpha}{1-\beta}\right)\left(\frac{\sqrt{2 z}}{2(1-\beta)}\right)^{-\alpha /(1-\beta)}\left(\begin{array}{c}
\sqrt{2 z} t^{1-\beta} J_{\frac{\alpha}{1-\beta}+1}\left(\frac{\sqrt{2 z}}{1-\beta} t^{1-\beta}\right) \\
J_{\frac{\alpha}{1-\beta}}\left(\frac{\sqrt{2 z}}{1-\beta} t^{1-\beta}\right)
\end{array}\right) \\
=\left(\begin{array}{c}
\frac{z}{\alpha+1-\beta} t^{\alpha+2-2 \beta}(1+o(1)) \\
t^{\alpha}(1+o(1))
\end{array}\right)
\end{gathered}
$$


и второй - дополнительный, компоненты которого станут коэффициентами при $\lambda$ (п. 3) леммы 1). Возьмем одно из следующих выражений в зависимости от значений $\alpha$ и $\beta$ :

$$
\begin{aligned}
& \Gamma\left(1-\frac{\alpha}{1-\beta}\right)\left(\frac{\sqrt{2 z}}{2(1-\beta)}\right)^{\alpha /(1-\beta)}\left(\begin{array}{c}
-\sqrt{2 z} t^{1-\beta} J_{\frac{-\alpha}{1-\beta}-1}\left(\frac{\sqrt{2 z}}{1-\beta} t^{1-\beta}\right) \\
J_{\frac{-\alpha}{1-\beta}}\left(\frac{\sqrt{2 z}}{1-\beta} t^{1-\beta}\right)
\end{array}\right) \\
& =\left(\begin{array}{c}
2 \alpha t^{-\alpha}(1+o(1)) \\
t^{-\alpha}(1+o(1))
\end{array}\right), \quad \frac{\alpha}{1-\beta} \notin \mathbb{N} \cup\{0\} ; \\
& \frac{-\left(\frac{\sqrt{2 z}}{2(1-\beta)}\right)^{\alpha /(1-\beta)}}{\Gamma\left(\frac{\alpha}{1-\beta}\right)} \\
& \times\left(\begin{array}{c}
\sqrt{2 z} t^{1-\beta}\left(\pi Y_{\frac{\alpha}{1-\beta}+1}\left(\frac{\sqrt{2 z}}{1-\beta} t^{1-\beta}\right)-2 \ln \frac{\sqrt{2 z}}{1-\beta} J_{\frac{\alpha}{1-\beta}+1}\left(\frac{\sqrt{2 z}}{1-\beta} t^{1-\beta}\right)\right) \\
\pi Y_{\frac{\alpha}{1-\beta}}\left(\frac{\sqrt{2 z}}{1-\beta} t^{1-\beta}\right)-2 \ln \frac{\sqrt{2 z}}{1-\beta} J_{\frac{\alpha}{1-\beta}}\left(\frac{\sqrt{2 z}}{1-\beta} t^{1-\beta}\right)
\end{array}\right) \\
& =\left(\begin{array}{c}
2 \alpha t^{-\alpha}(1+o(1)) \\
t^{-\alpha}(1+o(1))
\end{array}\right), \quad \frac{\alpha}{1-\beta} \in \mathbb{N} \\
& \frac{1}{2(1-\beta)}\left(\begin{array}{c}
\sqrt{2 z} t^{1-\beta}\left(\pi Y_{1}\left(\frac{\sqrt{2 z}}{1-\beta} t^{1-\beta}\right)-2 \ln \frac{\sqrt{2 z}}{1-\beta} J_{1}\left(\frac{\sqrt{2 z}}{1-\beta} t^{1-\beta}\right)\right) \\
\pi Y_{0}\left(\frac{\sqrt{2 z}}{1-\beta} t^{1-\beta}\right)-2 \ln \frac{\sqrt{2 z}}{1-\beta} J_{0}\left(\frac{\sqrt{2 z}}{1-\beta} t^{1-\beta}\right)
\end{array}\right) \\
& =\left(\begin{array}{c}
1+o(1) \\
\ln t(1+o(1))
\end{array}\right), \quad \alpha=0 \text {. }
\end{aligned}
$$

По поводу формул для решений-столбцов надо сделать несколько замечаний. Вид и коэффициенты в них подобраны так, что при переходе к пределу $z \rightarrow 0$ не возникает особенностей или вырождений, более того, есть непрерывность по $z$. Кроме того, вьводы о сушествовании решений с такой асимптотикой можно сделать непосредственно из коэффициентов уравнения (21), даже если не иметь точной формулы для этих решений. Выбор предельного решения также делается исходя из асимптотического поведения.

Получаемое из леммы 4 неравенство

$$
\left|y_{2}\left(\frac{N_{2}}{N}\right)\right|=\left|F_{N_{2}}\right|<k\left(\frac{N_{2}}{N}\right)^{2-2 \beta}
$$

позволяет оценить $\lambda$ для $y_{2}$. В самом деле, оценка для $\lambda$ получается из оценки для $y$ путем несложных рассуждений:

$$
\begin{aligned}
y(t) & =\frac{M_{11}(t)+\lambda M_{12}(t)}{M_{21}(t)+\lambda M_{22}(t)} \Longleftrightarrow \lambda=\frac{M_{11}(t)-y(t) M_{21}(t)}{y(t) M_{22}(t)-M_{12}(t)} \\
& \Longrightarrow|\lambda|<\frac{\left|M_{11}(t)\right|+|y(t)|\left|M_{21}(t)\right|}{\left|M_{12}(t)\right|-|y(t)|\left|M_{22}(t)\right|}, \text { если знаменатель }>0 .
\end{aligned}
$$


Таким образом, рассмотрев асимптотику решений, получим

$$
\begin{array}{ll}
|\lambda|<\varepsilon_{2}^{2(\alpha+1-\beta)} \frac{|z /(\alpha+1-\beta)|+k+o(1)}{2|\alpha|-k \varepsilon_{2}^{2-2 \beta}+o(1)} & \text { при } \quad \alpha \neq 0, \\
|\lambda|<\varepsilon_{2}^{2-2 \beta} \frac{|z /(1-\beta)|+k+o(1)}{1-k \varepsilon_{2}^{2-2 \beta}\left|\ln \varepsilon_{2}\right|+o(1)} & \text { при } \quad \alpha=0 .
\end{array}
$$

Итак, мы можем утверждать: если решение $y_{2}(t)$ уравнения $(21)$, построенного по $\varepsilon_{2}$, записать в виде (13), то параметр $\lambda$ при больших $N$ и малых $\varepsilon_{2}$ ограничен по формуле $(25)$ величиной, стремящейся к 0 при $\varepsilon_{2} \rightarrow 0$. Рассмотрим теперь решение дифференциального уравнения (21), соответствующее $\lambda=0$. Обозначим его $y_{0}$, попутно заметив, что именно это решение выделяется как $h_{z}$ краевым условием из формулировки замечания 1 к теореме 1 . Устремим $\varepsilon_{2}$ к нулю, зафиксировав $\varepsilon_{1}$. Тогда получим, что

$$
\varlimsup_{N \rightarrow \infty} \sup _{t \in\left[\varepsilon_{1}, 1\right]} \rho\left(y_{2}(t), y_{0}(t)\right)
$$

меньше сколь угодно малой величины. Вместе с (22), (23) это нам даст

$$
\varlimsup_{N \rightarrow \infty} \sup _{n \in\left[N_{1}, N\right]} \rho\left(y_{0}\left(\frac{n}{N}\right), F_{n}\right)=0 .
$$

Подставив $\lambda=0$ в (13) и используя формулу (24), получим точное выражение для $y_{0}(t)$ :

$$
y_{0}(t)=\sqrt{2 z} t^{1-\beta} J_{\frac{\alpha}{1-\beta}+1}\left(\frac{\sqrt{2 z}}{1-\beta} t^{1-\beta}\right) / J_{\frac{\alpha}{1-\beta}}\left(\frac{\sqrt{2 z}}{1-\beta} t^{1-\beta}\right) .
$$

Осталось подставить $n=N$, что соответствует $t=1$. Таким образом, теорема 1 доказана, как и формула (7). Докажем теперь следствие.

Теорема 1 (как и теорема А) дает возможность находить асимптотику нулей ортогональных многочленов в окрестности точки $x_{0}$ (в данном случае 0 ), но из-за свойства сходиться в сферической метрике это требует дополнительных рассуждений. Во-первых, $q_{n+1}$ и $q_{n}$ не могут иметь общих нулей, поэтому на любом компакте $K \subset \mathbb{C}$, не содержашем точек $\left\{z_{i}\right\}, J_{\frac{\alpha}{1-\beta}}\left(\frac{\sqrt{2 z_{i}}}{1-\beta}\right)=0$, по теореме 1 при достаточно большом $n$ отношение $q_{n}\left(c n^{2 \beta-2} z\right) / q_{n+1}\left(c n^{2 \beta-2} z\right)$ ограничено, т.е. $q_{n}\left(c n^{2 \beta-2} z\right) \neq 0$. Наоборот, для произвольно малой фиксированной окрестности $z_{i}$ из теоремы 1 следует, что величина

$$
\frac{q_{n}\left(c n^{2 \beta-2} z\right) q_{n+1}(0)}{n\left(q_{n+1}\left(c n^{2 \beta-2} z\right) q_{n}(0)-q_{n}\left(c n^{2 \beta-2} z\right) q_{n+1}(0)\right)}=\frac{1}{f_{n}(z)} \rightrightarrows \frac{1}{f(z)}
$$

и по теореме Руше в этой окрестности будет ровно 1 нуль левой части (26), т.е. ровно 1 корень $q_{n}\left(c n^{2 \beta-2} z\right)$. 


\section{§4. Доказательство теоремы 2}

Основные шаги и обозначения для доказательства теоремы 2 уже были сделаны при доказательстве теоремы 1: мы ввели величины $r_{n}-$ см. $(9)$, и там же было указано, что при подстановке $x=0$ в соотношение (4) можно выразить рекуррентньй коэффициент $\Delta_{n}$. Условие (5) задает поведение $r_{n}$. Получаем:

$$
\frac{b_{n-1} b_{n}}{\Delta_{n}}=\frac{\left(1+r_{n-1}\right)\left(1+r_{n}\right)}{r_{n}}, \quad r_{n}=1-\frac{2 \alpha+1}{n}+o\left(\frac{1}{n}\right)
$$

Теперь утверждение теоремы 2 получается в результате тождественных преобразований и простого суммирования:

$$
\begin{aligned}
\sum_{k=n}^{\infty} & \left(\frac{b_{k-1} b_{k}}{\Delta_{k}}-4\right)=\sum_{k=n}^{\infty}\left(\left(1+r_{k-1}\right)\left(1+r_{k}^{-1}\right)-4\right) \\
= & \sum_{k=n}^{\infty}\left[\left(r_{k-1}-r_{k-1}^{-1}\right)-\left(r_{k}-r_{k}^{-1}\right)\right] \\
& \quad+\sum_{k=n}^{\infty} \frac{r_{k-1}}{r_{k}}\left(1-\frac{r_{k}}{r_{k-1}}\right)^{2}+\sum_{k=n}^{\infty}\left(r_{k}-1\right)\left(1-r_{k-1}^{-1}\right) \\
= & \left(r_{n-1}-r_{n-1}^{-1}\right)+\sum_{k=n}^{\infty} o\left(\frac{1}{k^{2}}\right)+\sum_{k=n}^{\infty} \frac{(2 \alpha+1)^{2}}{k(k+1)}+o\left(\frac{1}{k^{2}}\right) \\
= & \frac{4 \alpha^{2}-1}{n}+o\left(\frac{1}{n}\right) .
\end{aligned}
$$

\section{§5. Примеры}

ПримеР 1. В качестве примера применения теоремы 1 для некомпактного носителя рассмотрим одну из классических систем - полиномы Лагерра. Исходными данньми служат рекуррентные коэффициенты и значения в точке 0 полиномов, нормированных по старшему коэффициенту. Отметим также, что при попытке взять любую другую точку в качестве начальной после сдвига, переводящего эту точку в 0, и пересчета $b_{n}$ мы увидим, что условие теоремы 2 не выполнено. Исходные данные для полиномов с параметром $p(p>-1)$ :

$$
b_{n}=2 n+p+1, \quad \Delta_{n}=n(n+p), \quad q_{n}(0)=(-1)^{n} \frac{\Gamma(n+p+1)}{\Gamma(p+1)} .
$$

Отсюда находим, что

$$
1+r_{n}=\frac{-b_{n} q_{n}(0)}{q_{n+1}(0)}=\frac{2 n+p+1}{n+p+1}=2-\frac{p+1}{n}+o\left(\frac{1}{n}\right) .
$$

Таким образом, параметры из теоремы 1 имеют следующие значения: $c=2$; $\beta=1 / 2 ; \alpha=p / 2$. Значит, асимптотика ближайших нулей будет такой:

$$
x_{k, n}=\frac{1}{4} j_{k}^{2}(p) n^{-1}(1+o(1)),
$$

где, напомним, через $j_{k}(s)$ обозначен $k$-й нуль функции Бесселя $J_{s}(x)$. Этот результат согласуется с асимптотическими формулами, приведенными, например, в книге Сегё [3]. 
Пример 2. В работе [2] при исследовании неравенства Бернштейна-Маркова для весов Гегенбауэра возникла необходимость получить асимптотику наименьшего нуля для системы ортогональных полиномов со следуюшими коэффициентами:

$$
\begin{gathered}
b_{n}=\frac{1}{2}\left(\frac{n+a}{(2 n+1)(2 n+a)(2 n+a+1)}+\frac{n}{(2 n+2 a-1)(2 n+a)(2 n+a-1)}\right) \\
\Delta_{n}=\frac{n(n+a-1)}{4(2 n-1)(2 n+a)(2 n+a-1)^{2}(2 n+a-2)(2 n+2 a-1)}
\end{gathered}
$$

Поскольку рекуррентные коэффициенты стремятся к нулю, то в данном случае мы имеем дело с чисто дискретной мерой. В той же работе были приведены и значения полиномов в нуле, поэтому у нас есть все необходимые начальные данные:

$$
q_{n}(0)=(-1)^{n} \frac{n ! \Gamma(n+a)}{(2 n) ! \Gamma(2 n+a)} .
$$

Проверяем выполнение условий теоремы 1 и находим значения параметров. Из формулы для $b_{n}$ находим, что $c=1 / 8 ; \beta=-1$. Для проверки последнего условия и нахождения $\alpha$ подсчитаем $r_{n}$ (это проще сделать по следующей формуле):

$$
r_{n}=\frac{\Delta_{n} q_{n-1}(0)}{q_{n+1}(0)}=\frac{n(2 n+1)(2 n+a+1)}{(n+a)(2 n+a-1)(2 n+2 a-1)}=1-\frac{2 a-2}{n}+o\left(\frac{1}{n}\right)
$$

Таким образом, последнее условие выполнено и $\alpha=a-3 / 2$. Кроме того, величины $q_{n}(0)$ все время меняют знак, а значит, все их корни положительны. Отсюда следует, что асимптотика наименьшего корня есть

$$
x_{1, n} \sim \frac{1}{4} j_{1}^{2}\left(\frac{2 a-3}{4}\right) n^{-4}
$$

ПримеР 3. Рассмотрим систему ортогональных многочленов со следующими рекуррентньми коэффициентами:

$$
b_{n}=0, \quad \Delta_{n}=\frac{(n+1)^{2}}{4(n+1)^{2}-1} .
$$

Эти коэффициенты суть сдвинутые на 1 коэффициенты полиномов Лежандра, и соответствующие им многочлены - это многочлены второго рода для системы Лежандра. Они имеют следуюшие значения в точке 1 (правый конец отрезка ортогональности):

$$
q_{n}(1)=2^{-n} \Gamma\left(\frac{3}{2}\right) \frac{\Gamma^{\prime}(n+2)+\gamma \Gamma(n+2)}{\Gamma(n+3 / 2)}, \quad \gamma-\text { константа Эйлера. }
$$

Этот пример интересен тем, что хотя мы и имеем дело с мерой на отрезке, но условия теоремы А не выполнены, и применить ее не удается. Действительно, найдем асимптотическое поведение отношения ортонормированных полиномов в точке 1 , 
выразив его через значения полиномов с единичным старшим коэффициентом. Получим следующий результат (через $\psi(x)$ обозначена логарифмическая производная гамма-функции):

$$
\begin{aligned}
\frac{q_{n+1}(1)}{q_{n}(1) \sqrt{\Delta_{n+1}}} & =\frac{n+2}{2 n+3} \frac{\psi(n+3)+\gamma}{\psi(n+2)+\gamma} \frac{\sqrt{4(n+2)^{2}-1}}{n+2} \\
& =1+\frac{1}{2 n}+\frac{1}{n(\ln n+\gamma)}+O\left(\frac{1}{n^{2}}\right) .
\end{aligned}
$$

Видно, что параметр $\alpha$ формально равен нулю (см. (2)), но остаточньй член не имеет вида $O\left(n^{-1-\varepsilon}\right), \varepsilon>0$. Это связано с тем, что теорема А описывает меры, имеющие (пусть в слабом смысле) чисто степенное поведение на конце отрезка ортогональности, а в нашем случае это не так. Явный вид меры ортогональности для этого случая может быть найден исходя из явного вида меры Лежандра, пользуясь связью (например, см. [4]) между преобразованием Коши меры ортогональности и рекуррентными коэффициентами. Обозначим через $\mu_{0}$ и $\mu$ меру Лежандра и нашу, а через $\widehat{\mu}_{0}$ и $\widehat{\mu}$ - соответственные преобразования Кошш. Имеем:

$$
\begin{gathered}
d \mu_{0}(x)=\frac{d x}{2} \text { на }[-1,1], \quad \widehat{\mu}_{0}(z)=\frac{1}{z-\widehat{\mu}(z) / 3}, \\
\widehat{\mu}(z)=3\left(2\left(\int_{-1}^{1} \frac{d x}{z-x}\right)^{-1}-z\right)=6 \ln ^{-1}\left(\frac{z+1}{z-1}\right)-3 z .
\end{gathered}
$$

Отсюда определяем меру через скачок $\widehat{\mu}(z)$ на вещественной оси. Получаем

$$
d \mu(x)=\frac{6 d x}{\ln ^{2}((1+x) /(1-x))+\pi^{2}} \quad \text { на }[-1,1]
$$

Такое логарифмическое зануление меры на концах не мешает, однако, применить теорему 1 и найти первый член асимптотики отношений соседних полиномов $q_{n}$ и асимптотику нулей $q_{n}$.

\section{§6. Расширения метода}

Для нахождения асимптотического поведения самих полиномов теорема 1 не обеспечивает достаточную точность, так как ряд из членов порядка $o(1 / n)$ может расходиться. Но методы, использованные в ее доказательстве, можно использовать и для нахождения следуюших членов асимптотики отношений, а отсюда можно получить результаты и для асимптотики полиномов. Покажем это на примepe 3 .

Рассматривая конструкцию доказательства теоремы 1 , мы видим, что оно состоит из двух этапов. На первом этапе мы оцениваем величину $F_{n}$ для "малых" $n$. На втором этапе мы подбираем векторное поле, трансляции вдоль которого близки к преобразованию $F_{n}$ “за один шаг” для "немалых" $n$. Если мы увеличим точность на каждом этапе, мы сможем увеличить точность в ответе. 
Итак, сделаем параллельньй перенос $x \rightarrow x-1$. Тогда конец интервала ортогональности перейдет в 0 и новые коэффициенты $b_{n}=-1$. Правая часть формулы (27) теперь является значениями полиномов в нуле, так что мы, как и ранњше, определяем $r_{n}$. Получаются следующие выражения:

$$
1+r_{n}=\frac{2 n+3}{n+2} \frac{\psi(n+2)+\gamma}{\psi(n+3)+\gamma}, \quad r_{n}=\frac{n+1}{n+2} \frac{\psi(n+1)+\gamma}{\psi(n+3)+\gamma}
$$

Изменим для удобства второе соотношение $(9)$, определяюшее $F_{n}$, и выпишем новый вариант соотношения (10):

$$
\begin{gathered}
\frac{q_{n}(x)}{q_{n-1}(x)}=\frac{q_{n}(0)}{q_{n-1}(0)}\left(1-\frac{F_{n}}{n+1}\right), \quad F_{1}=\frac{2 x}{b_{0}} \\
\frac{F_{n+1}}{n+2}=\frac{x}{b_{n}}\left(1+r_{n}\right)+r_{n} \frac{F_{n}}{n+1}\left(1-\frac{F_{n}}{n+1}\right)^{-1} \quad(n>0) .
\end{gathered}
$$

Пусть теперь $A_{n}$ - последовательность вида const $\cdot r_{1}^{-1} \cdots r_{n-1}^{-1}$ или близкая к ней.

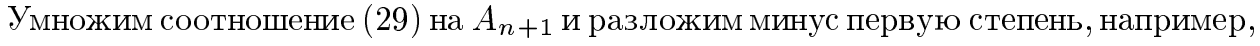
дважды. Преобразуя, получим:

$$
\begin{aligned}
\frac{F_{n+1}}{n+2} A_{n+1}-\frac{F_{n}}{n+1} A_{n} & =\frac{x}{b_{n}} A_{n+1}\left(1+r_{n}\right)+\left(A_{n+1} r_{n}-A_{n}\right) \frac{F_{n}}{n+1} \\
& +r_{n}\left(\frac{F_{n}}{n+1}\right)^{2} A_{n+1}+r_{n}\left(\frac{F_{n}}{n+1}\right)^{3}\left(1-\frac{F_{n}}{n+1}\right)^{-1} A_{n+1} .
\end{aligned}
$$

Подставим сюда $x=-z N^{-2}, A_{n}=(n+1)(\psi(n+1)+\gamma)(\psi(n+2)+\gamma)$ и $(28)$ :

$$
\begin{aligned}
& F_{n+1}(\psi(n+2)+\gamma)(\psi(n+3)+\gamma)-F_{n}(\psi(n+1)+\gamma)(\psi(n+2)+\gamma) \\
& =z N^{-2}(2 n+3)(\psi(n+2)+\gamma)^{2} \\
& \quad+\left[\left(\frac{F_{n}}{n+1}\right)^{2}+\left(\frac{F_{n}}{n+1}\right)^{3}\left(1-\frac{F_{n}}{n+1}\right)^{-1}\right](n+1)(\psi(n+1)+\gamma)(\psi(n+2)+\gamma) .
\end{aligned}
$$

Складывая по $n$ и учитывая, что лемма 4 для этого примера справедлива, получаем сперва уточняющий ее результат:

$$
\begin{aligned}
& F_{n+1}(\psi(n+2)+\gamma)(\psi(n+3)+\gamma) \\
& \quad=z N^{-2}\left(\frac{1}{2}(n+2)^{2}[(\psi(n+2)+\gamma)(\psi(n+3)+\gamma)\right. \\
& \quad+(\psi(n+2)+\gamma-1)(\psi(n+3)+\gamma-1)]+\mathrm{const})+O\left(\frac{n^{4} \ln ^{2} n}{N^{4}}\right) .
\end{aligned}
$$

Отметим, что невозможно найти константу по асимптотическим данным, так как, изменив начало последовательности рекуррентных коэффициентов, можно сделать так, что при $n$ больше некоторого $n_{0}$ значения $q_{n}(0)$ останутся неизменньми, 
а значения $q_{n}^{\prime}(0)$ изменятся. Но в данном случае можно найти константу, используя значение $F_{1}$. Таким образом, получим формулу

$F_{n}=(n+1)^{2} z N^{-2}\left[\frac{1}{2}+\frac{1}{2}\left(1-\frac{1}{\psi(n+1)+\gamma}\right)\left(1-\frac{1}{\psi(n+2)+\gamma}\right)\right]+O\left(\frac{n^{4}}{N^{4}}\right)$.

Эту формулу, в свою очередь, можно использовать повторно, получив после суммирования выражение

$$
\begin{aligned}
& F_{n}=(n+1)^{2} z N^{-2}\left[\frac{1}{2}+\frac{1}{2}\left(1-\frac{1}{\psi(n+1)+\gamma}\right)\left(1-\frac{1}{\psi(n+2)+\gamma}\right)\right] \\
& +\frac{n^{2}(n+1)^{2} z^{2}}{4 N^{4}(\psi(n+2)+\gamma)}\left[\psi(n+1)+\gamma-\frac{5}{2}+\frac{21}{8(\psi(n+1)+\gamma)}-\frac{1}{(\psi(n+1)+\gamma)^{2}}\right] \\
& +\frac{n^{6} z^{3}}{12 N^{6}}+O\left(\frac{n^{2}}{N^{4} \ln n}\right)+O\left(\frac{n^{6}}{N^{6} \ln n}\right) .
\end{aligned}
$$

Соотношение (29) после подстановок выражений для $r_{n}, b_{n}$ и $x$ выглядит так:

$$
F_{n+1}=(2 n+3) z N^{-2} \frac{\psi(n+2)+\gamma}{\psi(n+3)+\gamma}+F_{n}\left(1-\frac{F_{n}}{n+1}\right)^{-1} \frac{\psi(n+1)+\gamma}{\psi(n+3)+\gamma}
$$

т.е. матрица дробно-линейного преобразования, нормированная по ее определителю, равна

$$
\sqrt{\frac{\psi(n+3)+\gamma}{\psi(n+1)+\gamma}}\left(\begin{array}{cc}
\frac{\psi(n+1)+\gamma}{\psi(n+3)+\gamma}-\frac{2 n+3}{n+1} \frac{\psi(n+2)+\gamma}{\psi(n+3)+\gamma} z N^{-2} & (2 n+3) \frac{\psi(n+2)+\gamma}{\psi(n+3)+\gamma} z N^{-2} \\
\frac{-1}{n+1} & 1
\end{array}\right) .
$$

Теперь нам надо подобрать матрицу “непрерывной трансляции", для которой матрица “дискретной трансляции” отличается от данной на малую величину. Это можно сделать, подбирая асимптотическое разложение по степеням $n$. При этом получаются довольно громоздкие выражения. $\mathrm{K}$ примеру, рассматривая члены до порядка $O\left(n^{-2-\varepsilon}\right)$, получим

$$
N\left(\begin{array}{cc}
-\frac{1}{n(\ln n+\gamma)}-\frac{z}{N^{2}}+\frac{\ln n+\gamma+1}{n^{2}(\ln n+\gamma)^{2}} & (2 n+2) \frac{z}{N^{2}} \\
-\frac{1}{n}+\frac{1}{2 n^{2}}-\frac{1}{n^{2}(\ln n+\gamma)} & \frac{1}{n(\ln n+\gamma)}+\frac{z}{N^{2}}-\frac{\ln n+\gamma+1}{n^{2}(\ln n+\gamma)^{2}}
\end{array}\right) .
$$

Поэтому уравнение, описьвающее эволюцию последовательности $F_{n}$ с точностью до $O\left(n^{-1-\varepsilon}\right)$, имеет вид

$$
\begin{aligned}
y^{\prime}= & \left(2 t+\frac{2}{N}\right) z-\left(\frac{2}{t(\ln N+\ln t+\gamma)}+\frac{2 z}{N}-\frac{2(\ln N+\ln t+\gamma+1)}{(\ln N+\ln t+\gamma) N t^{2}}\right) y \\
& +\left(\frac{1}{t}+\frac{1-(\ln N+\ln t+\gamma) / 2}{N t^{2}(\ln N+\ln t+\gamma)}\right) y^{2}
\end{aligned}
$$

Предельное уравнение при $N \rightarrow \infty$ будет

$$
y^{\prime}=2 t z+y^{2} / t
$$


и оно фигурирует в теореме 1 . Но использовать его в качестве начального приближения, рассматривая (32) как возмущенное уравнение, плохо, так как порядок возмущения есть $\ln ^{-1} N$ и ответ будет в виде разложения по степеням $\ln ^{-1} N$. Чтобы иметь возможность выписывать ряд по $1 / N$, нужно решать уравнение

$$
y^{\prime}=2 t z-\frac{2 y}{t(\ln N+\ln t+\gamma)}+\frac{y^{2}}{t} .
$$

K счастью, оно тоже решается, причем его решения просто связаны с решениями (33). Эта связь не имеет случайного характера, а обусловлена следующим: поскольку обычные полиномы Лежандра $P_{n}$ имеют те же рекуррентные коэффициенты (после сдвига $n$ ), то дискретные преобразования для отношений $P_{n+1} / P_{n}$ совпадают с рассматриваемым случаем; в то же время для значений полиномов Лежандра величины $r_{n}$ таковы, что уравнение (33) уже дает точность $O(1 / N)$. Сведение возможно уже в терминах $F_{n}$. Приведем иллюстрацию:

$$
\begin{gathered}
\text { формула }(31) \text { и } \frac{\psi(n+2)+\gamma}{\psi(n+1)+\gamma}\left(1-\frac{F_{n}}{n+1}\right)=1-\frac{G_{n}}{n+1} \\
\Rightarrow G_{n+1}=(2 n+3) z N^{-2}+G_{n}\left(1-\frac{G_{n}}{n+1}\right)^{-1} .
\end{gathered}
$$

Таким образом, все сложности, связанные с появлением разных логарифмических членов в разложениях, локализованы в “начальных условиях" (формула (30) уточнение леммы 4$)$ и в соотношении между $F_{n}$ и $G_{n}:(\psi(n+1)+\gamma) G_{n}+1=$ $(\psi(n+2)+\gamma) F_{n}$. Матрица дискретных преобразований для величин $G_{n}$ и соответствующая матрица непрерывных преобразований будут следующими $(n \leqslant N)$ :

$$
\begin{gathered}
\left(\begin{array}{cc}
1-\frac{2 n+3}{n+1} z N^{-2} & (2 n+3) z N^{-2} \\
-\frac{1}{n+1} & 1
\end{array}\right), \\
N\left(\begin{array}{cc}
-z N^{-2} & (2 n+2) z N^{-2} \\
-\frac{1}{n+1 / 2} & z N^{-2}
\end{array}\right)+O\left(\frac{N}{n^{3}}\right) .
\end{gathered}
$$

Матрица непрерывных преобразований дает уравнение Риккати вида

$$
y^{\prime}=2\left(t+\frac{1}{N}\right) z-\frac{2 z}{N} y+\frac{y^{2}}{t+1 /(2 N)}
$$

с точностью $O\left(N^{-2}\right)$. Его решение с той же точностью будет

$$
\frac{z t}{N}+\left(t+\frac{1}{2 N}\right) \sqrt{2 z} \frac{J_{1}([t+1 /(2 N)] \sqrt{2 z})+\lambda Y_{1}([t+1 /(2 N)] \sqrt{2 z})}{J_{0}([t+1 /(2 N)] \sqrt{2 z})+\lambda Y_{0}([t+1 /(2 N)] \sqrt{2 z})}, \quad \lambda=\text { const }
$$

а сдвиг на $1 / N$ имеет ошибку $O\left(n^{-3}\right)$. Возьмем $n=N_{0}$ порядка $N^{3 / 4}$ и используем формулу (30) для выбора $\lambda$. Обозначим через $\rho(n)$ ошшбку в $G_{n}$. Тогда, как и 
в доказательстве теоремы 1 , из лемм 2,3 и неравенства треугольника получаем рекурсивные оценки на ошибку:

$$
\begin{gathered}
\rho\left(N_{0}\right)=O\left(N^{-3 / 2}\right), \quad \rho(n+1) \leqslant \frac{n+1}{n} \exp \left(\frac{C}{N}\right) \rho(n)+O\left(n^{-3}\right) \\
\left(N \geqslant n \geqslant N_{0} \sim N^{3 / 4}\right) .
\end{gathered}
$$

Это вариант неравенства Гронуолла, и отсюда стандартным образом получим явную оценку на ошибку:

$$
\rho(n) \leqslant \exp \left(\frac{C n}{N}\right) n\left(O\left(N^{-9 / 4}\right)+\sum_{N_{0}}^{n} O\left(k^{-4}\right)\right), \quad \rho(N)=O\left(N^{-5 / 4}\right) .
$$

Расчет $\lambda$ дает результат

$$
\lambda=-\frac{\pi}{\ln \left(z /\left(2 N^{2}\right)\right)}+O\left(N^{-5 / 4}\right)
$$

Теперь мы можем локализовать корни полиномов $q_{n}$ лучше, чем утверждает теорема 1. С учетом сделанной вначале трансляции имеем: если $q_{N}\left(1-z N^{-2}\right)=0$, то $F_{N+1}=\infty$, т.е. $G_{N+1}=\infty$, т.е. вьполнено следующее условие:

$$
\ln \left(\frac{z}{2 N^{2}}\right) J_{0}\left(\sqrt{2 z}\left(1+\frac{3}{2 N}\right)\right)=\pi Y_{0}\left(\sqrt{2 z}\left(1+\frac{3}{2 N}\right)\right)+O\left(N^{-5 / 4} \ln N\right) .
$$

На основании соотношения (37) можно получить асимптотические выражения для нулей полиномов Лежандра второго рода, заменяя функции Бесселя $J_{0}$ и $Y_{0}$ для аргументов, близких к $j_{k}(0)$, на степенные ряды. В этих выражениях будут участвовать константы $j_{k}(0), Y_{0}\left(j_{k}(0)\right), J_{1}\left(j_{k}(0)\right), Y_{1}\left(j_{k}(0)\right)$. Но условие $(35)$ точнее, чем все такие выражения, так как оно определяет $z$ с точностью до $O\left(N^{-5 / 4}\right)$, а любое выражение, полученное по производньм $J_{0}$ и $Y_{0}$, будет иметь точность $O\left(\ln ^{-m} N\right)$.

Переходим к нахождению асимптотики самих полиномов, а не их отношений. Соотношение, определяющее $F$, можно переписать в следующем виде:

$$
\frac{q_{n}(x)}{q_{n}(0)}=\frac{q_{n-1}(x)}{q_{n-1}(0)}\left(1-\frac{F_{n}}{n+1}\right)
$$

откуда

$$
\begin{gathered}
q_{n}(x)=q_{n}(0) \prod_{m=1}^{n}\left(1-\frac{F_{m}}{m+1}\right) \\
q_{N}(c z N-2)=q_{N}(0) \frac{\psi\left(N_{0}+1\right)+\gamma}{\psi(N+2)+\gamma} \prod_{m=1}^{N_{0}-1}\left(1-\frac{F_{m}}{m+1}\right) \prod_{m=N_{0}}^{N}\left(1-\frac{G_{m}}{m+1}\right) .
\end{gathered}
$$

Теперь считаем первое произведение, используя оценку (30), а второе произведение - используя (35), (34) и (36). В результате получим следующую формулу:

$$
\begin{aligned}
q_{N}\left(c z N^{-2}\right)= & \frac{q_{N}(0)}{\psi(N+2)+\gamma}\left(J_{0}\left(\frac{N+1 / 2}{N} \sqrt{2 z}\right)\right. \\
& \left.\quad-\frac{\pi}{\ln \left(z /\left(2 N^{2}\right)\right)} Y_{0}\left(\frac{N+1 / 2}{N} \sqrt{2 z}\right)+O\left(N^{-5 / 4}\right)\right) .
\end{aligned}
$$


Вспомним теперь, что для приведения рекуррентных соотношений к виду теоремы 1 мы делали сдвиг на 1. Вернув переменную $x$ и выполнив обратный сдвиг, получаем следующую асимптотическую формулу для полиномов Лежандра второго рода:

$$
\begin{aligned}
q_{N}(1-x)=2^{-N} & \frac{\Gamma(3 / 2) \Gamma(N+2)}{\Gamma(N+3 / 2)}\left(J_{0}\left(\left(N+\frac{1}{2}\right) \sqrt{2 x}\right)\right. \\
& \left.-\frac{\pi}{\ln (x / 2)} Y_{0}\left(\left(N+\frac{1}{2}\right) \sqrt{2 x}\right)+O\left(N^{-5 / 4}\right)\right),
\end{aligned}
$$

где $N^{2} x \in K$. Анализируя последние вькладки, можно заметить, что таким путем удается доказать асимптотическую формулу для значений самих полиномов только в предположении, что в (34) знаменатель не обрашается в нуль. На самом деле формула справедлива и без такого ограничения. Чтобы обойти указанную трудность, нужно переходить через участки номеров $n$, соответствующие окрестностям "плохих" значений $t$, вычисляя $q_{n}$ опосредованно, по такой схеме: $q_{n}$ удовлетворяют линейному разностному уравнению, поэтому их можно представить в виде линейной комбинации других решений того же уравнения. Рассматривая отношения соседних членов любого решения разностного уравнения и определяя аналоги величин $F_{n}$, мы получим для них то же рекуррентное соотношение, что и для $F_{n}$. Поэтому их можно приблизить решениями того же уравнения Риккати, но с другими $\lambda$. Взяв два значения $\lambda^{(1)}, \lambda^{(2)}$, для которых знаменатель в (34) отделен от нуля в некоторой окрестности “плохого" значения $t_{0}$, получим два решения $G_{n}^{(1)}, G_{n}^{(2)}$ и будем иметь

$$
\prod^{n}\left(1-\frac{G_{m}}{m+1}\right)=C_{1} \prod^{n}\left(1-\frac{G_{m}^{(1)}}{m+1}\right)+C_{2} \prod^{n}\left(1-\frac{G_{m}^{(2)}}{m+1}\right)
$$

Тогда слева от $N t_{0}$ асимптотика левой части доказана, что позволяет найти $C_{1}$ и $C_{2}$. Для значений $n / N$ в окрестности $t_{0}$ считаются произведения, содержащие $G^{(1)}$ и $G^{(2)}$. Таким образом можно показать, что формулы для произведения остаются истинными и после значения $t_{0}$.

\section{Список литературы}

1. Anтекарев А. И. Асимптотика ортогональных многочленов в окрестности концов интервала ортогональности // Матем. сб. 1992. Т. 183. № 5. С. 43-62.

2. Draux A., Elhami Ch. On the positivity of some bilinear functionals in Sobolev spaces // J. Comput. Appl. Math. 1999. V. 106. № 2. P. 203-243.

3. Сегё Г. Ортогональные многочлены. М.: Физматгиз, 1961.

4. Ахиезер Н. И. Классическая проблема моментов. М.: Физматгиз, 1961.

Нижегородский государственный технический университет

E-mail : root@waise.nntu.sci-nnov.ru
Поступила в редакцию 24.01 .2000 и 15.12 .2000 